\title{
Kinetically Assisted Potential Sputtering of Insulators by Highly Charged Ions
}

\author{
G. Hayderer, S. Cernusca, M. Schmid, P. Varga, HP. Winter, and F. Aumayr* \\ Institut für Allgemeine Physik, TU Wien, Wiedner Hauptstrasse 8-10, A-1040 Vienna, Austria \\ D. Niemann, V. Hoffmann, and N. Stolterfoht \\ Hahn Meitner Institut, Glienickerstrasse 100, D-10109 Berlin, Germany \\ C. Lemell, L. Wirtz, and J. Burgdörfer \\ Institute for Theoretical Physics, Vienna University of Technology, \\ Wiedner Hauptstrasse 8-10, A-1040 Vienna, Austria
}

(Received 12 September 2000)

\begin{abstract}
A new form of potential sputtering has been found for impact of slow ( $\leq 1500 \mathrm{eV})$ multiply charged Xe ions (charge states up to $q=25$ ) on $\mathrm{MgO}_{x}$. In contrast to alkali-halide or $\mathrm{SiO}_{2}$ surfaces this mechanism requires the simultaneous presence of electronic excitation of the target material and of a kinetically formed collision cascade within the target in order to initiate the sputtering process. This kinetically assisted potential sputtering mechanism has been identified to be present for other insulating surfaces as well.
\end{abstract}

PACS numbers: 34.50.Dy, 79.20.Rf

Ever since intense sources for slow, highly charged ions (HCI) have become available two decades ago, the possibility of exploiting the huge amount of potential energy stored in these projectiles for surface modification and nanofabrication has captured the imagination of researchers. Applications have been envisioned ranging from information storage via material processing to biotechnology. Compared to kinetic sputtering (i.e., sputtering of target atoms due to momentum transfer in a collision cascade), which unavoidably causes radiation damage in deeper layers, sputtering induced by the potential energy of slow highly charged ions [termed potential sputtering (PS)] holds great promise as a tool for more gentle nanostructuring. A profound understanding of the mechanisms responsible for conversion of projectile potential energy in PS processes is therefore highly desirable.

PS phenomena have been reported by several groups for a variety of insulator target surfaces as, e.g., alkali halides [1,2], $\mathrm{SiO}_{2}$ [3], $\mathrm{UO}_{x}$ [4], GaAs [5], mica [6], and hydrocarbon contaminated surfaces [7,8]. All investigations have in common that a dramatic increase of the total sputter yields, the secondary ion emission yields, or the size of single ion-induced defects with increasing projectile charge state has been observed. Depending on the surface material and/or the charge state and impact energy of the projectiles, several complementary models have been suggested to explain PS. The "Coulomb explosion" model $[9,10]$ has long been favored, but with the exception of proton sputtering from hydrocarbon covered surfaces $[8,11]$ has failed to provide even a semiquantitative interpretation of experimental data [12]. For GaAs a model to explain the observed high sputtering yields [5] was recently suggested, which involves structural instabilities arising from the destabilization of atomic bonds due to a high density of electronic excitation [13] produced during the neutral- ization and penetration of very highly charged ions with typically $500 \mathrm{keV}$ where the kinetic energy exceeds the available potential energy.

For slow medium charge-state projectile ions $(q \leq 27)$ on alkali halides and $\mathrm{SiO}_{2}$ the so-called "defect-mediated desorption" model has been most successful [12] in describing the experimental data $[1,3,14]$. This model requires a target material with strong electron-phonon coupling, where electronic excitations can be localized by forming lattice defects via self-trapping [e.g., "self-trapped excitons" (STE) and "self-trapped holes" (STH)] [15]. The decay of these defects in the near surface region into color centers then gives rise to the desorption of mostly neutral atoms. Very recently, the prediction of the defect-mediated desorption model for the onset of sputtering has been verified for $\mathrm{LiF}$ [2]. By using various singly charged projectiles the minimum potential energy necessary to induce PS from LiF was determined to be about $10 \mathrm{eV}$. This threshold just coincides with the potential energy necessary to produce a "cold hole" (the precursor of a STH) in the valence band of LiF by resonant neutralization of the ion $[2,16]$. This result provided evidence for the defectmediated sputtering mechanism to be operative in materials with strong electron-phonon coupling.

In order to identify further insulators which are also subject to PS we have extended our investigations to $\mathrm{Al}_{2} \mathrm{O}_{3}$ and $\mathrm{MgO}$ targets. State-of-the-art recipes and techniques have been used for preparation and characterization of these target films. $\mathrm{MgO}$ and $\mathrm{Al}_{2} \mathrm{O}_{3}$ layers have been produced by $e$-beam evaporation of the respective powder at an oxygen pressure of about $10^{-3}$ mbar. One has to be aware of the fact that $\mathrm{MgO}$ films produced in this way always contain $\mathrm{MgO}_{2}$ enriched surface layers [17]. And, indeed, x-ray photoelectron spectra of our samples exhibited a broad peak at the position of $\mathrm{MgO}$ with a shoulder 
towards higher energies which was identified as the signature of a $\mathrm{MgO}_{2}$ enriched surface layer [17]. This is why we refer to our target in the following as $\mathrm{MgO}_{x}$.

Self-trapping, as required by the defect-mediated desorption model, is known to occur in $\mathrm{Al}_{2} \mathrm{O}_{3}$ but not in highly ionic oxides like $\mathrm{MgO}_{x}$. Consequently, we expected to observe PS only for $\mathrm{Al}_{2} \mathrm{O}_{3}$ targets, especially since earlier experiments with $\mathrm{Ar}^{q+}(q \leq 8)$ on $\mathrm{MgO}$ did not indicate PS. It came as a big surprise that a strong charge-state effect was present when we first bombarded $\mathrm{MgO}_{x}$ with multiply charged $\mathrm{Xe}$ ions (charge states up to $q=25$ ). The unusual behavior of the measured total sputtering yields for $\mathrm{MgO}_{x}$ with projectile impact velocity, however, leads us to the conclusion that we have encountered a new form of PS.

The experiment was performed using the $14.5-\mathrm{GHz}$ electron cyclotron resonance source at the Ionenstrahl-Labor of the Hahn-Meitner-Institut in Berlin. The ion source provides Xe projectiles in sufficient quantities (nA) with charge states up to $q=28$. The end of the beam line is equipped with a deceleration lens system to obtain ions with impact energies as low as $5 \times q \mathrm{eV}$. For our measurements only ions with total kinetic energies between 100 and $1500 \mathrm{eV}$ have been used. To study HCI-induced potential sputtering a quartz crystal microbalance technique has been applied [18]. The target material is first deposited on the quartz crystal as a thin polycrystalline film and then bombarded by ions of interest. The sputter yield is determined from the frequency shift of the quartz crystal due to the mass loss of the target film. Our technique is able to detect mass changes of $0.5 \%$ of a monolayer per minute [18] on the quartz crystal microbalance which is thermally stabilized at a temperature of about $200{ }^{\circ} \mathrm{C}$. All experiments were made in UHV at a residual gas pressure of about $10^{-10}$ mbar.

In Fig. 1 the mass removal (in atomic mass units per incident ion, as determined by our quartz crystal microbalance technique) due to the impact of $\mathrm{Xe}^{q+}$ ions $(q=9$, $14,19,25,28)$ on $\mathrm{Al}_{2} \mathrm{O}_{3}$ is plotted as a function of ion impact energy. Corresponding results for $\mathrm{Xe}^{q+}(q=9,14$, 19, 23, 25) on $\mathrm{MgO}_{x}$ are given in Fig. 2. The data shown are values as obtained from freshly prepared surfaces. For both targets a dependence of the sputtering yield on total ion dose was found. For $1 \mathrm{keV} \mathrm{Xe} \mathrm{X}^{14+}$ ions, e.g., the sputtering yield of $\mathrm{Al}_{2} \mathrm{O}_{3}$ already drastically decreased at an ion dose of $2 \times 10^{13}$ ions $/ \mathrm{cm}^{2}$. The integrated mass loss at this total dose corresponds approximately to the removal of all oxygen atoms from the first monolayer of $\mathrm{Al}_{2} \mathrm{O}_{3}$. For PS from $\mathrm{SiO}_{2}$ a surface decomposition due to preferential desorption of oxygen and the formation of a $\mathrm{Si}$ overlayer leading to reduced sputtering has already been demonstrated [3,14]. For the $\mathrm{MgO}_{x}$ target a much higher ion dose, corresponding to the ablation of about ten monolayers, had to be applied before a decrease in sputtering yield became noticeable (cf. Fig. 3). For both target species the original sputter values could, however, be re-

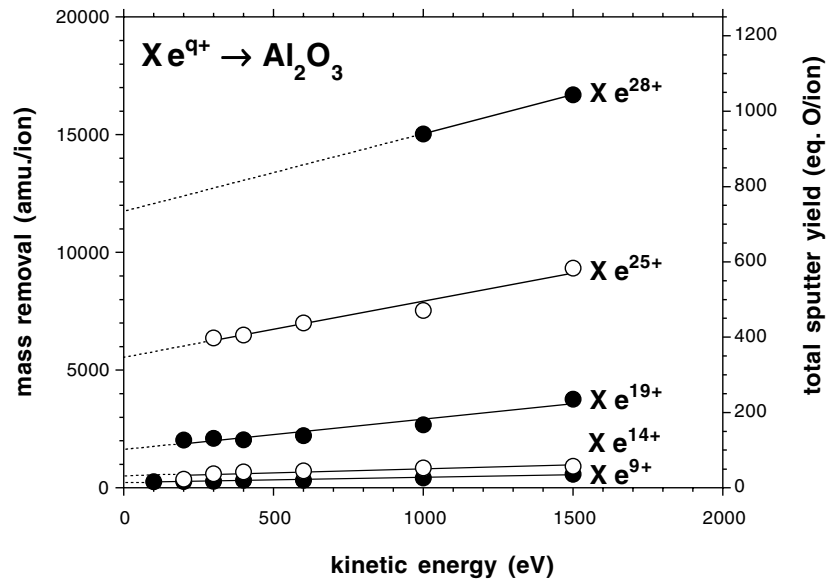

FIG. 1. Mass removal due to sputtering of $\mathrm{Al}_{2} \mathrm{O}_{3}$ by highly charged $\mathrm{Xe}^{q+}$ ions $(q=9,14,19,25$, and 28) as a function of ion impact energy. Left ordinate: in atomic mass units per incident projectile (as measured by the quartz crystal microbalance). Right ordinate: corresponding sputter yield (in $\mathrm{O}$ atoms per incident ion) under the assumption that only $\mathrm{O}$ atoms are sputtered. Solid lines for guidance only; dashed lines: extrapolation to zero kinetic energy.

stored by reoxidation of the samples in air. Since postoxidation of $\mathrm{MgO}$ films leads to $\mathrm{MgO}_{2}$ enriched surface layers [17] the observed dose dependence is interpreted as the transition between (a rather thick) oxygen enriched surface layer and bulk $\mathrm{MgO}$. We are, of course, aware that our data for $\mathrm{Xe}^{q+}$ ions on $\mathrm{MgO}_{x}$ presented in Fig. 2 are not consistent with earlier sputter yield data for $\mathrm{Ar}^{q+}$ on $\mathrm{MgO}$ [14]. However, these preliminary data were taken with an insufficiently characterized sample prepared in a different way (evaporation of $\mathrm{MgO}$ in ambient oxygen at lower pressure and with higher rate). Moreover, at that time our quartz crystal microbalance was not yet as sensitive

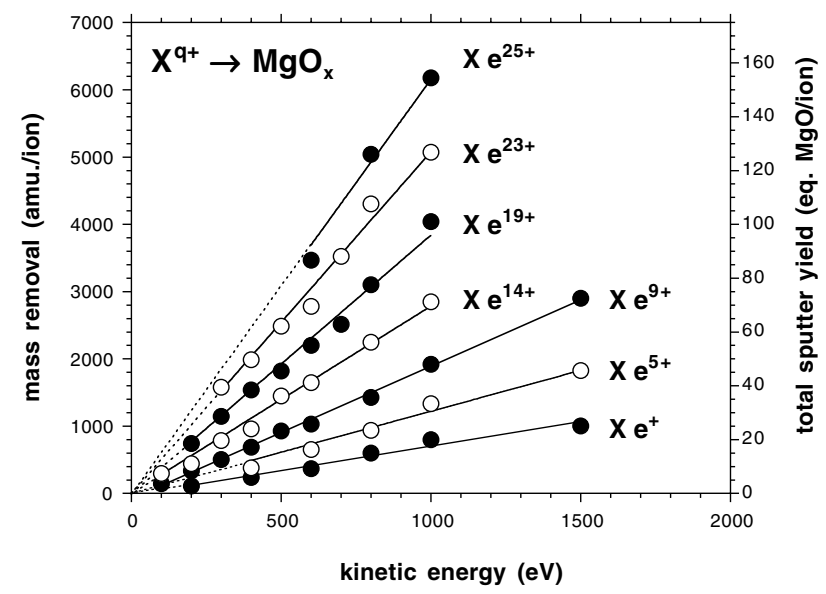

FIG. 2. Mass removal due to sputtering of $\mathrm{MgO}_{x}$ by highly charged $\mathrm{Xe}^{q+}$ ions $(q=1,5,9,14,19,23$, and 25) as a function of ion impact energy. Left ordinate: in atomic mass units per incident projectile (as measured by the quartz crystal microbalance). Right ordinate: corresponding sputter yield (in $\mathrm{MgO}$ molecules per incident ion). Solid lines for guidance only; dashed lines: extrapolation to zero kinetic energy. 


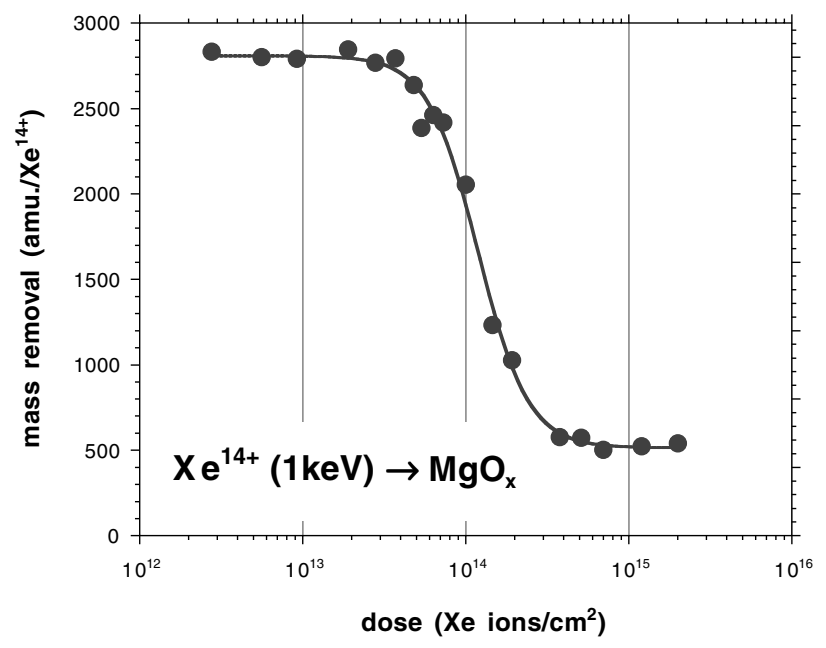

FIG. 3. Mass removal due to sputtering of $\mathrm{MgO}_{x}$ by $\mathrm{Xe}^{14+}$ ions (impact energy $1 \mathrm{keV}$ ) as a function of total ion dose. Solid curves for guidance only.

as it is now, and data could be obtained only after the target film had been bombarded with ions of a considerably higher dose, introducing additional uncertainties (surface structure and composition, dose dependent yield; cf. Fig. 3). Because of too low count rates, no systematic study of the charge-state dependence and reproducibility could be made at that time.

For $\mathrm{Al}_{2} \mathrm{O}_{3}$ the dependence of the measured sputtering yield on projectile charge state and impact velocity closely resembles the results earlier obtained for $\mathrm{LiF}$ and $\mathrm{SiO}_{2}$ (i.e., strong increase with projectile charge state, weak dependence on projectile kinetic energy, and thus high yields in the extrapolated limit of zero kinetic energy) [3], consistent with predictions of the defect-mediated desorption model. Consequently, $\mathrm{Al}_{2} \mathrm{O}_{3}$ can be considered another, technologically interesting candidate for surface modification by slow $\mathrm{HCI}$ (beside $\mathrm{SiO}_{2}$ and the alkali halides).

$\mathrm{MgO}_{x}$, however, exhibits an unusually strong dependence on the ion kinetic energy. Although the potential energy greatly enhances the total sputtering yield $Y$ (yield is proportional to the potential energy $E_{\text {pot }}$ ), it does not seem to be sufficient to induce PS on its own. Extrapolation of $Y$ to zero kinetic energy for all charge states is consistent with zero sputtering yield. Only in combination with projectile kinetic energy conspicuously large sputtering yields are achieved.

We interpret this surprising behavior as a new form of potential sputtering which requires simultaneously the electronic excitation of the target material (believed to be the precursor of the usual PS process) and the formation of a collision cascade within the target (and therefore a finite projectile kinetic energy) in order to initiate the sputtering process. This new mechanism may be termed "kinetically assisted potential sputtering." In the following we will sketch a model for this new mechanism, which combines our knowledge about the neutralization of slow HCI upon surface impact with that for radiation induced processes in nonmetallic solids [19]. According to the common scenario for highly charged ion-surface interaction [20] the potential energy of a HCI is deposited in the target surface via a series of electronic transitions (resonant electron transitions and Auger processes). These transitions lead to electronic excitation of a small surface region, i.e., create electron-hole pairs in the valence band and inner shell holes of the target. As a precursor for a potentialenergy induced sputtering process localization of the electronic excitation is required in order to effectively transfer the electronic energy into kinetic energy of the atomic and molecular particles to be desorbed. One important mechanism for pinning of the electronic excitations is trapping at lattice defects. In insulating solids with strong electronphonon coupling a strong lattice distortion gives rise to self-trapping (STE, STH formation). In other materials a localization of electronic excitation energy can occur only at already present defects [19] created by other processes or at interfaces. It is therefore plausible to assume that the kinetic energy of the projectile via a collision cascade may be responsible for generating the seed for the trapping of electronic excitations. For impact of several $100 \mathrm{eV}$ Xe projectile, elastic collision processes - even below the knock-on threshold for sputtering - can lead to a strong temporary displacement of lattice atoms and therefore provide sites for localization of electronic excitation energy. This scenario can be translated into a set of coupled rate equations for the sputtering yield $Y$ valid for any insulating crystal,

$$
\begin{aligned}
\frac{d Y}{d t}= & c_{P} N_{\mathrm{ST}}+c_{\mathrm{KP}} N_{\mathrm{LD}} N_{\mathrm{ED}} \\
& + \text { terms of higher order in } N_{X},
\end{aligned}
$$

where $c_{P}$ describes the conversion rate of a self-trapped electronic defect into desorption of surface particles (i.e., potential sputtering), $c_{\mathrm{KP}}$ the corresponding conversion rate of a pair of electronic and kinetically induced lattice defects. Analogous rate equations for $N_{\mathrm{ST}}$ (number of self-trapped electronic defects), $N_{\mathrm{LD}}$ (number of lattice defects), and $N_{\mathrm{ED}}$ (number of electronic defects) close the system. In the case of $\mathrm{MgO}_{x} c_{P}$ is zero signifying the absence of self-trapping, while for target materials that feature self-trapped electronic defects $\left(\mathrm{LiF}, \mathrm{SiO}_{2}, \mathrm{Al}_{2} \mathrm{O}_{3}\right)$ a nonzero value of $c_{P}$ is responsible for the measured sputtering yield at zero kinetic energy (cf. Fig. 1 and [1-3]). Since SRIM-2000 simulations [21] indicate that $N_{\mathrm{LD}}$ is proportional to the kinetic energy of the projectile, the second term in Eq. (1) gives rise to a sputtering yield $Y$ which increases linearly with kinetic energy and where the slope is a function of charge state $q$ due to the increased number of electronic defects $N_{\mathrm{ED}}$ for projectiles with higher $q$.

While some of the free parameters in Eq. (1) can be taken from the literature or simulations, a complete determination is not possible. We therefore use Eq. (1) 
in reverse: we fit the parameters to the data and test for consistency with known predictions. This allows us to draw some conclusions as to the underlying processes. For example, for an ideal $\mathrm{MgO}$ single crystal the measured high sputtering yield $Y$ would lead to values of $c_{\mathrm{KP}}$ far beyond accepted values for kinetically induced defects. According to simulations using SRIM-2000 the lattice binding energy would have to be smaller than $1 \mathrm{eV}$ to allow for the production of a sufficient number of lattice defects to explain the experimental data. The accepted threshold value for a perfect $\mathrm{MgO}$ crystal is, however, $\geq 20 \mathrm{eV}$. The sputtering data provide therefore, apart from the x-ray photoelectron spectroscopy (XPS) data, independent evidence for the presence of a very weakly bound $\mathrm{MgO}_{2}$ layer still covering the surface. While we could not find any information on the electronic or lattice structure of $\mathrm{MgO}_{2}$ surface layers, we can infer from our data that the threshold for kinetically induced defects must be small $(<1 \mathrm{eV})$. Projectiles then produce a large number of lattice defects $N_{\mathrm{LD}}$ along their trajectory in the target serving as possible trapping sites. At these sites the large number of electronic defects produced by highly charged $\mathrm{Xe}^{q+}$ may get localized resulting in the surprisingly large sputtering yields observed in our experiment.

This new form of kinetically assisted potential sputtering (KAPS) [second term in Eq. (1)] should, however, also be observable for target materials where self-trapping is possible (cases with $c_{P} \neq 0$ ). And, indeed, a closer inspection of Fig. 1 (data for $\mathrm{Al}_{2} \mathrm{O}_{3}$ ) and our earlier data for $\mathrm{LiF}$ and $\mathrm{SiO}_{2}$ (see, e.g., Figs. 1 and 2 from Ref. [3], respectively) reveals that also in these cases $Y$ increases linearly for increasing kinetic energy with the slope being a steep function of the charge state $q$. This behavior cannot be explained by conventional kinetic sputtering and has not been recognized in the past, since it is overshadowed by the comparably much stronger contribution from desorption due to self-trapped defects [first term in Eq. (1)].

The identification of a heretoforth unrecognized kinetically assisted potential sputtering process is therefore not based on the results for $\mathrm{MgO}_{x}$ alone (although these data did provide the first clue that a considerably more complex behavior was at hand than believed earlier). The KAPS mechanism seems to be present in a larger variety of target materials and might also provide an explanation for some projectile charge-state dependent sputtering and secondary ion emission phenomena observed at considerably higher kinetic energies (see [22] and references therein). The KAPS mechanism considerably expands the opportunities to modify surfaces using beams of slow highly charged ions.

This work has been supported by Austrian Fonds zur Förderung der Wissenschaftlichen Forschung. The authors thank Wolfgang Werner for performing the XPS measurements.

*To whom correspondence should be addressed. Email address: aumayr@iap.tuwien.ac.at

[1] T. Neidhart et al., Phys. Rev. Lett. 74, 5280 (1995).

[2] G. Hayderer et al., Phys. Rev. Lett. 83, 3948 (1999).

[3] M. Sporn et al., Phys. Rev. Lett. 79, 945 (1997).

[4] T. Schenkel et al., Phys. Rev. Lett. 80, 4325 (1998).

[5] T. Schenkel et al., Phys. Rev. Lett. 81, 2590 (1998).

[6] D. Schneider et al., Surf. Sci. 294, 403 (1993).

[7] S. Della-Negra et al., Phys. Rev. Lett. 60, 948 (1988).

[8] N. Kakutani et al., Nucl. Instrum. Methods Phys. Res., Sect. B 96, 541 (1995).

[9] I. S. Bitensky and E. S. Parilis, J. Phys. (Paris) C 2, 227 (1989).

[10] H.P. Cheng and J.D. Gillaspy, Phys. Rev. B 55, 2628 (1997).

[11] J. Burgdörfer and Y. Yamazaki, Phys. Rev. A 54, 4140 (1996).

[12] F. Aumayr et al., Comments At. Mol. Phys. 34, 201 (1999).

[13] P. Stampfli, Nucl. Instrum. Methods Phys. Res., Sect. B 107, 138 (1996).

[14] P. Varga et al., Phys. Scr. T73, 307 (1997).

[15] K.S. Song and R.T. Williams, Self-Trapped Excitons, Springer Series in Solid-State Sciences Vol. 105 (SpringerVerlag, Berlin, 1993), and references therein.

[16] L. Wirtz et al., Surf. Sci. 451, 197 (2000).

[17] J. Wollschläger et al., Appl. Surf. Sci. 142, 129 (1999).

[18] G. Hayderer et al., Rev. Sci. Instrum. 70, 3696 (1999).

[19] N. Itoh, Nucl. Instrum. Methods Phys. Res., Sect. B 135, 175 (1998), and references therein.

[20] J. Burgdörfer et al., Phys. Rev. A 44, 5647 (1991); J. Burgdörfer, in Fundamental Processes and Applications of Atoms and Ions, edited by C. D. Lin (World Scientific, Singapore, 1993); A. Arnau et al., Surf. Sci. Rep. 27, 113 (1997); HP. Winter and F. Aumayr, J. Phys. B 32, R39 (1999).

[21] J. F. Ziegler et al., The Stopping and Range of Ions in Solids, edited by J.F. Ziegler (Pergamon Press, New York, 1985).

[22] T. Schenkel et al., Prog. Surf. Sci. 61, 23 (1999). 